\title{
Factors affecting shoot and root apical meristem tissue culture of Thai supersweet corn (Zea mays var. rugosa)
}

\begin{abstract}
During last recent years, in vitro propagation technic is widely used to produce plants with desirable traits. This experiment was conducted to produce an ideal protocol for in vitro propagation of Thai supersweet corn by using shoot apical meristem (SAM) and root apical meristem (RAM) as explants. Four-day-old germinating seedlings were used as the experimental materials on culture media supplemented with a range of auxin, kinetin, and carbohydrates. The primary establishment for SAM showed the highest percentage of survival (80\%) while RAM showed the highest survival (67\%) and in Murashige and Skoog (MS) media supplemented. Upon acclimatization, regenerated plantlets from shoot showed the highest survival rate (12\%) with the production of 21 plantlets; however, the survival rate of plantlets from root was only $20 \%$ with the production of 9 plantlets. The efficient and economic protocol that is produced in this study can be applied as an alternative to conventional propagation method for the large-scale production of Thai supersweet corn throughout the year.
\end{abstract}

Keyword: Shoot apical meristem; Root apical meristem; Thai supersweet corn; In vitro propagation; Tissue culture; Plant growth regulators 